\title{
As dificuldades na construção do modo de atenção psicossocial em serviços extra-hospitalares de saúde mental
}

\author{
Difficulties in developing psychosocial care in extra-hospital services providing mental \\ health care
}

Regina Célia Fiorati', Toyoko Saeki²

1 Doutora em Ciências pela Universidade de São Paulo (USP) - São Paulo (SP), Brasil. Docente do Curso de Terapia Ocupacional da Faculdade de Medicina de Ribeirão Preto, da Universidade de São Paulo (USP) - São Paulo (SP), Brasil.

reginacf@fmrp.usp.br

${ }^{2}$ Doutora em Enfermagem pela Universidade de São Paulo (USP) - São Paulo (SP), Brasil. Docente da Escola de Enfermagem de Ribeirão Preto, da Universidade de São Paulo (USP) - São Paulo (SP), Brasil.

maryto@eerp.usp.br
RESUMO Pretende-se avaliar de que forma o modo de atenção psicossocial tem sido construído e efetivado em serviços extra-hospitalares de saúde mental, como o Ambulatório Regional de Saúde Mental de Ribeirão Preto e o Centro de Atenção Psicossocial II, do Município de Ribeirão Preto, São Paulo. Entrevistas semidiretivas e grupos focais foram realizados com 22 profissionais da saúde. O referencial teórico e a análise dos dados apoiaram-se no referencial hermenêutico crítico. Observou-se alta tecnificação das ações de saúde, centralidade da tecnologia médico-clínica em relação a outras ações terapêuticas, com ênfase na patologia e medicamento, com a secundarização de ações psicossociais; e um processo de precarização das relações de trabalho. Aponta-se a necessidade de realização de pesquisa avaliativa participativa com objetivo de criar mecanismos que favoreçam a melhoria da assistência prestada com base no modo de atenção psicossocial.

PALAVRAS CHAVE: Saúde Mental; Serviços Hospitalares; Apoio Social; Serviços de Reabilitação.

ABSTRACT The aim of this study was to evaluate how psychosocial care has been developed and put into effect in extra-hospital services providing mental health care, such as the Mental Health Regional Outpatient Service and the Psychosocial Care Center II, both in Ribeirão Preto, SP, Brazil. Semi-structured interviews and focal groups were conducted with 22 health workers. The theoretical framework and data analysis were based on the critical hermeneutic approach. We observed highly technique-oriented actions, a focus on medical-clinical technology at the expense of other therapeutic actions, with an emphasis on the pathology and medication scheme while low priority was given to psychosocial actions in the midst of an ongoing deterioration in labor relations. There is a need to conduct participatory evaluative studies in order to create mechanisms to improve the delivery of care based on the psychosocial care model.

KEYWORDS: Mental Health; Extra-Hospital Mental Health Services; Psychosocial Care, Psychosocial Rehabilitation. 


\section{Introdução}

O modo de atenção psicossocial nasceu para substituir os hospitais psiquiátricos por um modelo assistencial comunitário. Tal modelo de atenção tem seu fundamento e sua organização a partir dos Movimentos de Reforma Psiquiátrica, que se iniciaram na Europa e nos Estados Unidos em fins da década de 1950 e incrementaram-se, principalmente, a partir de 1960. No Brasil, esses movimentos iniciaram-se na década de 1970, conhecendo seu auge nas décadas de 1980 e 1990. Como equipamentos de atenção psicossocial, encontram-se os serviços extra-hospitalares do Sistema Único de Saúde (SUS), tais como os primeiros Núcleos de Atenção Psicossocial (NAPS), os Centros de Atenção Psicossocial (CAPS), Centros de Convivência, Hospitais-Dia, residências terapêuticas, ambulatórios, programas de suporte social, defesa e promoção dos direitos humanos dos pacientes e familiares (AMARANTE, 1995; COSTA-ROSA, 2000).

O CAPS foi criado com a proposta de trabalhar com uma equipe multiprofissional. Seu número de atividades deveria ser amplo o bastante para englobar, além do atendimento medicamentoso, oficinas terapêuticas e de criação, e, ainda, atividades físicas e lúdicas. Além disso, a família deveria estar inserida em todo o contexto de tratamento do paciente (MIELKE et al, 2009).

Atualmente, os serviços extra-hospitalares de saúde mental formam uma rede importante na atenção psiquiátrica reestruturada. Tais serviços compóem as diretrizes centrais da política de saúde mental no Brasil, que são apontadas pelo Ministério da Saúde e orientadas pela perspectiva da reforma psiquiátrica brasileira (BRASIL, 2004).

Segundo Amarante (2007), essas diretrizes podem ser resumidas nas iniciativas de redução progressiva e gradual dos leitos em hospitais psiquiátricos, na garantia de assistência aos pacientes egressos dos hospitais e na criação e sustentação da rede extra-hospitalar. Além disso, segundo as orientaçóes da política de saúde mental brasileira, algumas noçốes devem adquirir relevância, tais como: a concepção de trabalho interdisciplinar; integralidade das ações de saúde; noçôes de acolhimento e escuta terapêutica; planos terapêuticos individualizados; reabilitação psicossocial e inclusão social; e açôes e parcerias construídas com ênfase em ações focadas na comunidade (BRASIL, 2004).

A realização de pesquisa em serviços extra-hospitalares de saúde mental constituiu uma ação importante para o estudo das formas atuais de organização sob as quais tais serviços vêm atuando, dos projetos terapêuticos e das ações terapêuticas disponibilizadas à clientela. Permite, ainda, avaliar de que forma a organização atual dos serviços vem contemplando os objetivos da reforma psiquiátrica brasileira, apesar das grandes diferenças no desenvolvimento e na consolidação dos serviços extrahospitalares de saúde mental, considerando-se as regióes e os municípios do País (BRASIL, 2007).

Dessa forma, o presente trabalho buscou conhecer de que forma os tratamentos na área de saúde mental têm contemplado, além da dimensão clínica, as açôes psicossociais que formam as bases das orientaçóes centrais preconizadas pela reforma psiquiátrica. E, assim, avaliar se os serviços extra-hospitalares de saúde mental têm substituído o modelo hospitalocêntrico por uma rede de atenção psicossocial com base na interpretação das concepçôes de profissionais envolvidos nesses serviços.

\section{Metodologia com base em abordagem quali- tativa e participativa}

A pesquisa foi realizada em dois serviços extra-hospitalares de saúde mental do Município de Ribeirão Preto, São Paulo: o Ambulatório Regional de Saúde Mental de Ribeirão Preto (ARSMRP) e o Centro de Atenção Psicossocial II (CAPS II), no período de 2007 a 2009. Ambos são serviços de referência em saúde mental nesse município e nessa região. O ARSMRP é o maior equipamento de saúde mental da cidade, enquanto que o CAPS II é o único serviço de saúde mental nessa modalidade e ocupa posição central na rede de serviços dessa especialidade.

O ARSMRP atende a uma população de $330 \mathrm{mil}$ habitantes oriundos das regióes norte, sul e leste do município, além de duas cidades da região, e realiza uma média de 8 mil atendimentos/ano. O CAPS II atende 
a uma população de 107 mil habitantes correspondentes à região do Distrito Central de Ribeirão Preto (SP). Este equipamento não disponibilizou censo sobre estimativa de atendimentos realizados por ano.

Importante destacar que, enquanto os CAPS provêm atenção a pessoas com transtornos mentais severos e persistentes, os ambulatórios de saúde mental dão suporte aos CAPS situados em território comum, atendendo aos casos de menor gravidade e realizando articulação com as equipes da Atenção Básica em Saúde. Entretanto, em cidades ou regiões nas quais não há um CAPS articulado ao ambulatório em um mesmo território, como no nosso estudo, o ambulatório deverá contemplar ação semelhante aos CAPS, atendendo aos usuários com quadros mais severos, bem como disponibilizando, além do tratamento clínico, reabilitação psicossocial (BRASIL, 2001; 2007). Por isso, neste trabalho, os dois serviços foram analisados conjuntamente, por cumprirem, nesse caso, a função de responderem a semelhantes necessidades de saúde, em seus territórios correspondentes.

Os sujeitos da pesquisa foram os profissionais diretamente ligados à assistência aos usuários. Estes formam os agentes responsáveis pela elaboração e implementação das propostas de trabalho e do projeto terapêutico do serviço. Os sujeitos constituíramse nas seguintes categorias: auxiliar de enfermagem, enfermeiro, psicólogo, médico psiquiatra, assistente social, terapeuta ocupacional, musicoterapeuta e gerentes (embora estes sejam médicos psiquiatras, na pesquisa, foram computados separadamente).

O estudo foi submetido à Secretaria Municipal de Saúde de Ribeirão Preto (SP) e ao Comitê de Ética em Pesquisa da Escola de Enfermagem de Ribeirão Preto, da Universidade de São Paulo, obtendo a sua aprovação sob protocolo no $0831 / 2007$. Todos os participantes assinaram previamente um Termo de Consentimento Livre e Esclarecido.

O percurso metodológico teve base em abordagem qualitativa, pois os elementos constituintes do estudo requeriam análise interpretativa, sendo de natureza ideológica, das construções históricas, da ordem do simbólico, remetendo a concepçóes e significados. Como técnicas de coleta de dados utilizaram-se entrevistas semidiretivas e grupos focais.

As entrevistas semidiretivas continham eixo organizado em três questóes:

1. Descreva o seu trabalho no serviço, objetivos, relacionando-o com os outros profissionais da equipe e com o projeto terapêutico.

2. Como é elaborado o projeto terapêutico do seu serviço.

3. Qual a sua concepção sobre a reabilitação psicossocial e descreva açóes psicossociais presentes em seu serviço.

As perguntas eram lidas juntamente com os participantes a partir de cópia impressa disponibilizada aos mesmos.

A metodologia considerou o processo de produção do conhecimento, construído juntamente com os profissionais da saúde. Para isto, foi utilizado o referencial hermenêutico-dialético de Jürgen Habermas, o qual preconiza a construção de projetos de açáo social baseados na interação dialógica entre homens e intersubjetivamente organizados de forma democrática por meio de uma simetria de posiçóes discursivas imbricadas (HABERMAS, 1988).

Foram realizadas 19 entrevistas com os seguintes profissionais: 4 auxiliares de enfermagem, 2 enfermeiras, 2 psicólogas, 2 terapeutas ocupacionais, 3 assistentes sociais, 1 musicoterapeuta, 3 psiquiatras e 2 gerentes.

Após a análise do conteúdo temático contido nos depoimentos, os dados foram agrupados em categorias analíticas. Dentre elas, as que se sobressaíram foram: o trabalho tecnificado e centrado no núcleo de competência especialista; a predominância da tecnologia médico-clínica sobre outras ações terapêuticas; a indefiniçâo que cercava o processo de elaboração e gestão do projeto terapêutico; precarização das relaçóes de trabalho; e a carência de açóes de reabilitação psicossocial.

Essas categorias foram apresentadas aos grupos focais e foi traçado um eixo centralizador para a discussão, que indagava por que, nos serviços, o tratamento disponibilizado baseava-se principalmente na ação médicopsiquiátrica, em detrimento da reabilitação psicossocial. 
Os 19 profissionais que participaram dos grupos focais, em sua maioria, foram os mesmos que participaram das entrevistas, com exceção de 2 auxiliares de enfermagem e 1 musicoterapeuta, que não estavam presentes no serviço, no dia da realização do grupo focal, e de 1 enfermeiro, 1 psicólogo e 1 psiquiatra, que foram incluídos nesta etapa.

A análise dos dados se deu com base na filosofia hermenêutica dialética de Jürgen Habermas, apoiada na interpretação e na compreensão das construçóes discursivas e concepçóes dos autores-sujeitos da pesquisa, e não uma análise apoiada na descrição de fatos e explicação de fenômenos (HABERMAS, 1987; 1988).

\section{Resultados e discussão dos dados: uma análise interpretativa reconstrutiva}

A análise dos dados nos serviços pesquisados mostrou os seguintes resultados: atenção com ênfase na tecnificação do trabalho e no centramento das açóes profissionais nos núcleos de competência, em detrimento de um campo interdisciplinar; predominância da tecnologia médico-clínica sobre outras açóes terapêuticas, com base na supervalorização da terapêutica medicamentosa e na subvalorização de ações de reabilitação psicossocial; ausência de uma organização entre as equipes no sentido de elaborarem projetos terapêuticos coletivos e interdisciplinares; e precarização das relaçóes de trabalho gerada no contexto das orientaçóes das políticas de saúde mental municipais. Além disso, percebeu-se que as concepçóes sobre a reabilitação psicossocial ressaltavam a ideia predominante de um processo de adaptação a condutas sociais normatizadas e como função externa aos serviços. Vejamos cada um dos pontos resultantes da pesquisa a seguir.

A tecnificação do trabalho nos serviços foi ressaltada por meio da percepçáo dos profissionais concentrados sobre o núcleo de competência de suas profissóes. Tal fato foi representado pelo conjunto de conhecimentos e técnicas que demarcam um determinado campo profissional.
[...] algumas atividades, você sendo um profissional, você tem a aprovação para fazer aquilo de acordo com sua formação. Por exemplo: um psicólogo chega aqui, ele já sabe que ele vai fazer psicoterapia; o médico chega aqui, ele já sabe que vai fazer um atendimento médico, porque tem uma estrutura já pré-conversada... É exatamente isso: o atendimento médico, atendimento psicoterápico, psicoterapia de apoio e terapia ocupacional - são profissionais habilitados pra fazerem o que estão aqui pra fazerem. (Entrevistado no 11 , serviço $n^{\circ} 1$ ).

No meu caso, eu tenho que fazer os atendimentos clínico-medicamentosos, então, eu acabo sendo focado para aquilo. Não tenho tempo pra pensar em outras coisas, fico focado naquilo que é estritamente necessário. (Entrevistado no 12 , serviço $n^{\circ} 2$ ).

Esse núcleo de competência contém, essencialmente, um conjunto de técnicas apreendidas como procedimentos constantes de intervenção frente a determinados eventos previamente estudados e classificados segundo critérios pré-estabelecidos. Nota-se que os profissionais se restringem à aplicação de técnicas que refletem sua especificidade profissional. Isto faz com que um campo interdisciplinar de atuação ampla apoiada em um projeto terapêutico da equipe náo seja construído. Consequência disso é a não integralidade das ações de saúde e o desconhecimento de situaçóes que a realidade empírica apresenta em determinados momentos como as necessidades específicas dos usuários referentes à organização de suas vidas cotidianas, diante das quais essas técnicas fechadas em seu núcleo se mostram insuficientes para uma resposta efetiva (CAMPOS, 2000a).

A formação profissional na área da saúde, voltada para o modelo biomédico e individualizante, herdeiro das instâncias da produção e estabelecimento do conhecimento positivo sobre processos de saúde-doença, produz técnicas precisas e objetivas voltadas para a abordagem do sujeito em seu processo de adoecimento (AYRES, 2001). Entretanto, o fenômeno de imposição de uma racionalidade instrumental e técnica sobre 
outra formada na interdisciplinaridade e na dialogicidade entre os participantes de um projeto de ação é percebido como um fenômeno cultural na sociedade contemporânea. Segundo Habermas (1987), a sociedade contemporânea é marcada pela imposição de uma racionalidade instrumental sobre outra comunicativa. A racionalidade instrumental, tecnificada sobre todos os aspectos, impede que acordos linguisticamente mediados sejam estabelecidos na produção de projetos sociais, destituindo a possibilidade da formação de consensos éticos, fundados na dialogicidade e em relaçôes democráticas entre os homens.

A cultura, apoiada em um processo de despolitização, é legitimada por uma consciência tecnocrática, sendo, ao mesmo tempo, uma auto-objetivação dos homens em categorias que fusionam a ação dirigida a fins, a instrumental, e os comportamentos adaptativos. Os modelos reificados da ciência invadem o mundo vivido sociocultural e adquirem um poder objetivo sobre a autocompreensão (WIGGERSHAUS, 2006).

Outro fato observado nos serviços pesquisados, e que também se mostra como consequência da discussão acima, é a organização do trabalho centrado na tecnologia médico-clínica, sendo predominante a reprodução da tecnologia médica como matriz técnico-ideológica. $\mathrm{O}$ tratamento disponibilizado aos usuários enfatiza as consultas médico-psiquiátricas, o diagnóstico, o tratamento psicopatológico e o seguimento medicamentoso. Portanto, o trabalho do profissional médico funciona como organizador central em relação aos outros profissionais não médicos, de tal forma que o tratamento médico é considerado dentro de uma dimensão de necessidade absoluta e imprescindível. Já as outras atividades terapêuticas são vistas em um campo de possibilidades, condicionadas a recursos materiais e humanos, mas não necessariamente presentes nos serviços. As falas observam isso:

Qual o nosso objetivo ao fazer a visita domiciliar? Ver se o paciente está seguindo o tratamento que, infelizmente, é em cima de um tratamento medicamentoso (Entrevistado $\mathrm{n}^{\circ}$ 6, serviço $\left.\mathrm{n}^{\circ} 1\right)$.
E essa coisa de triagem também cansa, você fica todo o tempo tentando convencer... A gente ficou 10, 12 anos tentando dizer pras coordenaçôes, pros médicos que era importante ter outros profissionais na triagem, que outros profissionais têm condiçôes, podem fazer, mas, sabe aquela coisa?, passar do modelo médico pra outra coisa. O modelo médico é muito forte, não só dentro dos médicos, mas em nós também, os profissionais de saúde, especialmente, os médicos, enfermeiros, que têm aquilo enraizado. (Entrevistado $n^{\circ} 2$, serviço no 2).

Nos serviços pesquisados há, portanto, uma sobreposição da racionalidade instrumental sobre a racionalidade prática. A racionalidade hegemônica na sociedade ocidental e que norteia as práticas médicas é a instrumental, pois fundamenta sua tecnologia no sistema científico de conhecimento positivo, das ciências biológicas, universais e generalizáveis, sob todos os aspectos, e constroem determinadas técnicas e instrumentos cuja finalidade é o êxito técnico da cura. Ao contrário, a reabilitação psicossocial se dá segundo uma racionalidade prática, ou seja, ocorre no terreno dos contextos das necessidades de saúde psicossociais dos usuários, que se relacionam a aspectos culturais, econômicos e políticos das relaçóes humanas.

Os profissionais dos serviços avaliados demonstraram que não há um projeto terapêutico com uma filosofia primordial norteadora das açóes e objetivos centrais do atendimento nesses locais. Percebe-se que as equipes têm se demitido da função de elaboração e gestão dos projetos terapêuticos e admitido orientaçóes prontas advindas dos órgãos gestores, municipais ou de outras esferas do poder, ou, ainda, guiado suas açóes profissionais com base nas disposiçóes técnicas e tecnologias próprias as suas especialidades.

Não acho que tenha um projeto terapêutico... Acho que é o que vem da secretaria. Já vem pronto e a gente vai organizando aqui... (Entrevistado no 3, serviço no 1 ). 
Nos já tivemos muitos projetos terapêuticos... Hoje, acho que cada um trabalha dentro de seu rol de atividades especificas de sua profissão. (Entrevistado no 10 , serviço no 2 ).

Entende-se por projeto terapêutico todo o plano que designa o modo operacional de uma determinada equipe de profissionais. Esse planejamento tem como referencial determinadas necessidades de uma clientela, as quais, cruzando com formas determinadas de compreensão de cuidado e atenção, vão gerar açôes em forma de trabalho. Ou seja, um projeto terapêutico é o conjunto de açóes que se configura como resultado da interface entre as ofertas tecnológicas dos profissionais (cuidado, saberes e instrumentos) e as necessidades de saúde dos usuários, refletindo uma determinada filosofia de trabalho (MERHY, 2000).

O projeto terapêutico pode ser avaliado como um tipo de consenso fundado, o qual foi descrito por Habermas (1988) para designar o processo de entendimento mútuo entre os homens. Este seria linguisticamente mediado com a finalidade de coordenação da ação social, obtido em processo de argumentação que permite aos participantes levar às últimas consequências o princípio da dúvida radical; e livre de quaisquer formas de coação e violência, caracterizado pela liberdade da crítica e do debate, que pode, inclusive, levar à revisão ou à superação de sistemas fundados em determinados marcos teóricos ou institucionais. E, ainda, que todos os participantes da discussão instaurada tenham chances simétricas com relação à escolha, à elaboração e ao proferimento dos discursos e na assunção de papéis em uma verdadeira situação dialógica. Segundo o autor, se essas condiçóes forem observadas, se assume metodologicamente um interesse emancipatório da espécie humana, isto é, uma situação comunicativa ideal geradora de consenso fundado verdadeiro, no qual todos os participantes sejam interessados, protagonistas, livres e iguais.

Assim, se pensarmos o projeto terapêutico como um tipo de consenso fundado, percebemos a necessidade dessa constituição dialógica enquanto terreno básico e norteador do processo de construção do projeto, segundo o marco teórico-prático do princípio da integralidade e das açóes ampliadas nos serviços de saúde mental. Para constituir-se tal situação dialógica, é necessário, contudo, que existam indivíduos atuando no papel de sujeitos agenciadores desse projeto. Para Campos (2000b), faz-se necessária a constituição de coletivos humanos democraticamente organizados e com a produção e o fortalecimento das subjetividades.

No entanto, outro fato deve ser considerado para a realização desse projeto, que é a precarização das relaçôes de trabalho. Nos serviços pesquisados, evidenciouse certo processo de sucateamento de suas condiçóes de funcionamento. Os espaços físicos não são adequados e, no caso do ambulatório, é inapropriado. Há carência prolongada e contínua de recursos materiais e humanos, e os profissionais veem-se frequentemente sobrecarregados, assumindo tarefas não diretamente ligadas as suas funçôes e realizando atividades de forma mecanizada, sem observar as reais necessidades de saúde e sociais da clientela. Não se observam, contudo, ações decorrentes das políticas de saúde mental do município que busquem uma resolubilidade da problemática evidenciada. Ao contrário, nota-se que tal situação foi gradativamente instaurada nos últimos anos como consequência da orientação das políticas de saúde mental do município, a qual parece estar alinhada a posiçóes favoráveis ao aumento de leitos hospitalares em detrimento do fortalecimento das equipes e da organização do trabalho nos serviços extra-hospitalares de saúde mental.

Um fato interessante ocorreu quando uma pequena parte dos profissionais que atuam nos serviços pesquisados e que pertenceram aos movimentos de reforma psiquiátrica conceberam a reabilitação psicossocial como dispositivo vital nos serviços e atentaram para sua desvalorização frente às açóes terapêuticas desenvolvidas. Entretanto, a maior parte dos profissionais, ingressada mais recentemente nos serviços, apresentou uma concepçáo de reabilitação psicossocial como método de aproximação do portador de sofrimento psíquico das condutas normatizadas e dos papéis sociais adequados, ou, em outros casos, como açóes que não integram o rol de funçôes dos serviços de saúde mental, devendo ser remetidas a outros equipamentos de atendimento que não sejam, especificamente, para tratamento das doenças mentais. 
Como consequência imediata, vê-se que açóes de reabilitação psicossocial não são realizadas nos serviços estudados, pois demandam orientação e investimento opostos aos que estão sendo valorizados e efetivados. A reabilitação psicossocial é uma abordagem que surge das novas propostas reordenadoras da atenção em psiquiatria após os movimentos de reforma psiquiátrica no mundo e no Brasil, e que se caracteriza por direcionar as ações de saúde, nos serviços extra-hospitalares de saúde mental, no sentido de aproximar as experiências existenciais decorrentes do adoecimento mental dos espaços físicos, socioculturais e de legitimidade cidadã da sociedade (BARROS et al, 2007).

\section{O modelo da atenção psicossocial: tratamento e inclusão social}

Para contemplar um projeto terapêutico interdisciplinar em saúde mental, hoje, é necessário apoiar-se em um modelo de atenção psicossocial que inclua ações de tratamento de caráter clínico e as de reabilitação psicossocial (ONOCKO-CAMPOS; FURTADO, 2006).

Sendo assim, aponta-se a necessidade de uma pesquisa avaliativa nesses serviços, coordenada por acadêmicos, órgáos científicos fomentadores de pesquisa e pelo Ministério da Saúde, com a participação dos agentes imbricados, como usuários, familiares, profissionais e gestores.

Essa avaliação poderá fornecer subsídios para a resolução de problemas relacionados à organização das atividades de cada profissional, com ênfase em mudanças nas abordagens conceituais e práticas, na melhoria da assistência aos usuários, na tomada de decisóes no setor e na transparência no uso de verbas públicas. Além disso, as avaliaçóes servem para a elaboração de programas e projetos, e como material de apoio às políticas públicas. Estas também podem ser utilizadas para o acompanhamento dos processos, resultados e impactos de determinadas açóes (TANAKA; MELLO, 2004; KIRSCHBAUM, 2009).

Ressalta-se a necessidade, contudo, da pesquisa avaliativa em saúde mental incluir metodologia qualitativa com base em abordagem participativa, pois, nesse campo, há forte presença de conteúdos simbólicos e políticos imbricados, além de possibilitar a inclusão e a responsabilização de todos os agentes inseridos e envolvidos nos serviços extra-hospitalares de saúde mental, maximizando as modificaçóes efetuadas a partir dos resultados (ALMEIDA;ESCOREL, 2001).

\section{Considerações Finais}

A realização dessa pesquisa mostrou como determinadas problemáticas têm-se colocado na base do processo de construção de um novo paradigma relacionado à concepção e à atenção em saúde mental, o modo de atenção psicossocial.

As formas de cuidado ainda refletem a terapêutica médico-clínica e medicamentosa, de caráter estritamente biológico e curativista, desvalorizando açôes de reabilitação psicossocial, cuja finalidade principal é a ressignificação existencial do adoecimento mental, a criação de novas formas de vida, a inclusão social e a promoção de saúde.

Assim, vimos que a implantação do modelo psicossocial encontra algumas barreiras, principalmente, no que diz respeito à elaboração e à gestáo de projetos terapêuticos interdisciplinares que visem à integralidade das açóes de saúde.

Os principais obstáculos, segundo os profissionais avaliados, originam-se das orientaçóes fornecidas pelas políticas públicas no campo da saúde mental e administrativas ao nível da esfera municipal, que destoam das preconizaçóes da Política Nacional de Saúde Mental. Estas últimas refletem os objetivos da reforma psiquiátrica brasileira, dando ênfase a um projeto de atenção psiquiátrica reformulada, com base no modelo de atenção psicossocial.

Nessa perspectiva, vê-se a necessidade do desenvolvimento de uma pesquisa avaliativa nos serviços extra-hospitalares de saúde mental do município de Ribeirão Preto (SP), buscando-se caminhos de resolubilidade para os impasses organizacionais e assistenciais. Além disso, é de extrema importância que se considere a visão dos diversos agentes imbricados nesses serviços, como usuários, familiares, profissionais e gestores, e que se possa propor transformações na assistência prestada, buscando a efetiva implantação do modelo de atenção psicossocial. 


\section{Referências}

ALMEIDA, P. F.; ESCOREL, S. Da avaliação em saúde à avaliação em Saúde Mental: gênese, aproximações teóricas e questões atuais. Saúde em Debate, Rio de Janeiro,v. 25, n. 58, p. 35-47, maio/ago. 2001.

AMARANTE, P. Loucos pela vida: a trajetória da Reforma Psiquiátrica no Brasil. Rio de Janeiro: SDE/ENSP, 1995.

AMARANTE, P. Saúde Mental e atenção psicossocial. Rio de Janeiro: FIOCRUZ, 2007.

AYRES, J. R. C. M. Sujeito, intersubjetividade e práticas de saúde. Ciência e Saúde Coletiva, Rio de Janeiro, v. 6, n. 1, p. 63-67, 2001.

BARROS, S. et al. Innovative practices for health care. Revista da Escola de Enfermagem da USP, São Paulo, n. 41, p. 815-819, 2007.

BRASIL. Ministério da Saúde. Secretaria Executiva. Secretaria de Atenção à Saúde. Legislação em Saúde Mental: 1990-2004. 5 ed. Brasília: Ministério da Saúde, 2004.

BRASIL. Ministério da Saúde. Secretaria de Atenção À Saúde. Relatório de Gestão 2003-2006: saúde mental no SUS: acesso ao tratamento e mudança do modelo de atenção. Brasília: Ministério da Saúde, 2007.

Presidência da República. Lei Federal no 10.216, 06 de abril de 2001. Disponível em: <http://www.planalto.gov.br/ccivil_03/ leis/LEIS_2001/L10216.htm>. Acesso em: 13 nov. 2012

CAMPOS, G. W. S. Saúde pública e saúde coletiva: campo e núcleo de saberes e práticas. Ciência e Saúde Coletiva. Rio de Janeiro, v. 5, n. 2, p. 219-230, 2000a.

Um método para análise e co-gestão de coletivos, a constituição do sujeito, a produção de valor de uso e a democracia em instituições: o método da roda. São Paulo: Hucitec, 2000b.
COSTA-ROSA, A. O modo psicossocial: um paradigma das praticas substitutivas ao modo asilar. In: AMRARANTE, P. (org). Ensaios, subjetividade, saúde mental, sociedade. Rio de Janeiro: FIOCRUZ, 2000. p. 141-168.

HABERMAS, J. Técnica e ciência como ideologia. Lisboa: Edições 70, 1987.

Teoría de La acción comunicativa. Madrid: Taurus, 1988.

KIRSCHBAUM, D. I. Nursing agents' perceptions on their work in mental health with psychotic patients in a psychosocial community center. Revista Latino Americana de Enfermagem, Ribeirão Preto, n.17, p. 368-373, 2009.

MERHY, E. E. Um ensaio sobre o médico e suas valises tecnológicas. Interface: Comunicação, Saúde, Educação, Botucatu, n. 4, p. 109-116, fev.2000.

MIELKE, F. B. et al. Mental care delivered in psychosocial care centers (CAPS) from the viewpoint of the professionals. Ciência e Saúde Coletiva, Rio de Janeiro, n. 14, p. 159-164, 2009.

ONOCKO-CAMPOS, R; FURTADO, J. P. Entre a saúde coletiva e a saúde mental: um instrumental tecnológico para avaliação de rede de Centros de Atenção Psicossocial (CAPS) do Sistema Único de Saúde. Cadernos de Saúde Pública, São Paulo,v. 22, n. 5, p. 10531062, maio 2006.

TANAKA, O. Y.; MELLO, C. Avaliação de programas de saúde do adolescente: um modo de fazer. São Paulo: EDUSP, 2004.

WIGGERSHAUS, R. A Escola de Frankfurt: história, desenvolvimento teórico, significação pública. Rio de Janeiro: DIFEL, 2006.

Recebido para publicação em Novembro/2012

Versão final em Junho/2013

Conflito de interesse: não houve

Suporte financeiro: inexistente 